\title{
A Fast Alternative to Computational Fluid Dynamics for High Quality Imaging of Blood Flow
}

\author{
Robert H.P. McGregor ${ }^{1}$, Dominik Szczerba ${ }^{1,2}$, Krishnamurthy Muralidhar ${ }^{3}$, \\ and Gábor Székely ${ }^{1}$ \\ 1 Computer Vision Laboratory, Sternwartstr. 7, 8092 Zürich, Switzerland \\ mcgregor@vision.ee.ethz.ch \\ 2 IT'IS Foundation for Research, Zürich, Switzerland \\ 3 Department of Mechanical Engineering, Indian Institute of Technology Kanpur, \\ Kanpur 208 016, UP, India
}

\begin{abstract}
Obtaining detailed, patient-specific blood flow information would be very useful in detecting and monitoring cardio-vascular diseases. Current approaches rely on computational fluid dynamics to achieve this; however, these are hardly usable in the daily clinical routine due to the required technical supervision and long computing times. We propose a fast measurement enhancement method that requires neither supervision nor long computation and it is the objective of this paper to evaluate its performance as compared to the state-of-the-art. To this end a large set of abdominal aortic bifurcation geometries was used to test this technique and the results were compared to measurements and numerical simulations. We find that this method is able to dramatically improve the quality of the measurement information, in particular the flow-derived quantities such as wall shear stress. Additionally, good estimation of unmeasurable quantities such as pressure can be provided. We demonstrate that this approach is a practical and clinically feasible alternative to fully-blown, time-consuming, patient-specific flow simulations.
\end{abstract}

\section{Introduction}

High quality imaging of blood flow patterns can provide useful information for understanding cardio-vascular pathologies, predicting their onset and choosing an optimal therapy strategy. There is particular interest in measuring wall shear stress (WSS) as this plays an important role in the development of atherosclerosis and other flow-related diseases such as aneurysms. Unfortunately WSS is extremely difficult to measure as it is related to the derivative of velocity close to the wall, precisely where most conventional imaging techniques are least reliable. The use of both phase contrast magnetic resonance imaging (PC-MRI) and Doppler ultrasound, which are able to resolve flow fields in vivo and noninvasively in complex geometries have been studied extensively (e.g. [1] or [2]). Although they are found to be well suited for estimating the approximate time 
and spatially-averaged WSS patterns, both these modalities suffer from poor resolution and, even more importantly, from inadequate accuracy near the wall due to partial volume effects or wall motion induced artifacts, thus making them unable to provide high quality WSS estimates. However, these limitations can effectively be overcome by using computational fluid dynamics (CFD) in conjunction with medical imaging [3], which allows for very detailed flow field representations. Moreover, CFD is able to provide any desired (and usually non-measurable) flow quantities such as vorticity or pressure distributions which can be very useful for, e.g., aneurysm rupture prediction. The richness of the CFD simulation data has motivated a large research effort to investigate its use in patient-specific flow imaging (see 4] for a review) and there is much hope that these results could significantly contribute to the improvement of the diagnosis and treatment of vascular diseases such as cerebral aneurysms [5]. However the use of computer simulations requires in-depth numerical knowledge and enormous computational power and time, which makes them impractical for routine patient care and has kept them out of the clinics for the time being.

We have already proposed a clinically feasible approach to the combination of patient-specific measurement data and CFD [6]. This previous work focussed on exploring the potential of such an enhancement technique on a simplified 2D case. We now evaluate the quality of such $3 \mathrm{D}$ flow field reconstructions using a large database of abdominal aortic bifurcation geometries. We focus in particular on the quality of the WSS estimations, as this type of secondary flow information is extremely sensitive to measurement accuracy. Acquisition of several hundred real patient cases is a dedicated effort in itself and is not the focus of our current study. We therefore use artificially generated but statistically meaningful geometries. This approach conveniently provides us with a ground truth to evaluate our results and simultaneously reduces artifacts caused by faulty reconstruction of the geometry. In practice, this measurement enhancement technique could be applied to any imaging modality which is able to detect the geometry and preferably some flow velocity data, but we have chosen to focus on PC-MRI as this can measure both geometry and time-resolved flow velocity fields non-invasively.

\section{Methods}

Enhancement method. The method is fully described in our previous work [6] and will just shortly be summarized here for completeness. We use a large database of pre-computed flow fields for a given anatomical location and use these to perform an interpolation of actually measured, sparse and noisy velocity data, allowing us to recover a patient-specific, high quality flow field. Principal component analysis is applied to the database and the enhancement is performed as an optimisation in frequency space, using the method proposed by Blanz and Vetter [7, analogously to the procedure used in statistical shape models. For a basic overview of this enhancement method see figure 1 The main difference to our previous 2D implementation lies in the mesh generation, mesh mapping and its associated coordinate transformation. Indeed, to achieve this in 3D with 


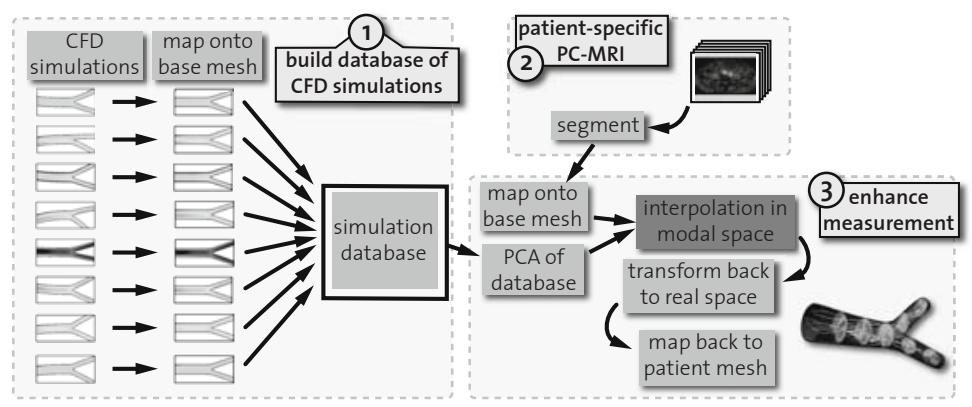

Fig. 1. Method flowchart

bifurcation geometries a radial coordinate system was used, the axial component being recovered by solving the Stokes equations with constant pressure boundary conditions at the inlet $(p 1=1)$ and both outlets $(p 2=0)$. In this way a coordinate system common to all bifurcations could be generated, thus making the velocity components comparable across geometries.

Building the database. As mentioned earlier, the database used here is built entirely from artificial data for evaluation purposes. A base mesh having the approximate physiological dimensions of an abdominal aortic bifurcation and being symmetric with respect to the sagittal plane was first generated. This was then deformed using 18 geometric parameters which were randomly varied within healthy physiological variability as described by Sun et al. [8] to create the various meshes. Additionally a random deformation field was applied to each geometry thus perturbing the vessel wall with high frequency local variations. Fifteen randomly selected bifurcations from the database can be seen in figure $4(\mathrm{f})$. Once the meshes became available, oscillatory flow simulations were performed in each one, using velocity boundary conditions at the inlet and fixed pressure at the outlet. Skewed parabolic velocity fields were used at the inlet, randomly varied with respect to oscillatory frequency, skewness and amplitude to mimic a variety of upstream conditions. The pressure was set to 0 at both outlets, using the assumption that the downstream conditions are the same for both iliac branches, which is physiologically reasonable. Each simulation was run for 3 cardiac cycles so as to reduce transient effects and the results from the last cycle were taken as our solutions. A total of 600 flow fields were generated in this fashion, each having its own specific geometry and boundary conditions.

Recovering the pressure fields. In addition to the velocities, the pressure fields were also stored in the database. This allows us to recover the patientspecific pressure distribution at very little extra computational cost, which can be interesting especially in the case of aneurysm rupture prediction, where the local pressure field plays an important role.

Evaluating the accuracy of the method. In order to evaluate our method, 100 of the flow fields were randomly selected for leave-one-out tests and therefore removed from the database. We then simulated PC-MRI measurements of each of these test cases. This was done in two steps. First we defined a typical voxel 
size of $1 \times 1 \times 4 \mathrm{~mm}$ and averaged the velocities inside each voxel for 20 equallyspaced timesteps. Secondly, we added white Gaussian noise with a maximal intensity of $5 \%$ of the velocity encoding value (VENC). At voxels which are on the boundary, the partial volume effect typical to PC-MRI was simulated by adding nodes with 0 value outside the geometry before averaging. We then compared the velocity, WSS and pressure distributions obtained from both the simulated flow measurements and from our enhancement method with the ground truth, given by the original simulations. WSS was computed in all cases by using quadratic shape functions of the tetrahedra having a face on the wall, so the acquisition data had first to be interpolated onto the mesh, similarly to Cheng et al. 9].

\section{Results}

Having applied the enhancement for 100 test cases, the most obvious way to present the results is a statistical evaluation: this can be found in table 1. For each quantity of interest and for each case we performed a linear regression analysis of the type seen in figures 2 and 3 to evaluate how well the measured and the enhanced 11 values compared to the simulated ones. We then considered the $r^{2}$ correlations (the square of Pearson's correlation coefficient) and the slope of the fitted linear functions as these best characterize the fit. The closer both these numbers are to 1 , the better the observation matches the known data. In addition to this statistical evaluation we show the simulated, measured and enhanced flow, WSS and pressure fields for a representative case in figure 4

Table 1. Mean values and standard deviations over the 100 test cases of the $r^{2}$ correlation coefficients and the slopes of the fitted linear curves relating both the measured and the enhanced data to the simulations (ground truth) for all components of velocity $(u, v, w)$, the norm of velocity $(|U|)$, the WSS and the pressure

\begin{tabular}{|l||c|c||c|c|}
\hline \multicolumn{1}{|c||}{} & \multicolumn{2}{c||}{ Measured } & \multicolumn{2}{c|}{ Enhanced } \\
\hline & $r^{2}$ & slope & $r^{2}$ & slope \\
\hline$u$ & $0.873 / 0.024$ & $0.818 / 0.028$ & $0.896 / 0.058$ & $0.897 / 0.051$ \\
$v$ & $0.897 / 0.017$ & $0.834 / 0.022$ & $0.917 / 0.053$ & $0.915 / 0.050$ \\
$w$ & $0.883 / 0.023$ & $0.876 / 0.018$ & $0.980 / 0.012$ & $0.971 / 0.014$ \\
$|U|$ & $0.842 / 0.029$ & $0.871 / 0.020$ & $0.976 / 0.013$ & $0.974 / 0.013$ \\
\hline$W S S$ & $0.824 / 0.023$ & $0.359 / 0.023$ & $0.947 / 0.059$ & $0.929 / 0.048$ \\
\hline pressure & & & $0.996 / 0.007$ & $0.938 / 0.131$ \\
\hline
\end{tabular}

Velocity. Figures 4(a) 4(b) and 4(c) show typical flow fields during end diastole. This phase of the heart cycle was chosen as the velocities are low and recirculations near the boundary take place. In all figures a detail of the streamlines

\footnotetext{
${ }^{1}$ We will systematically refer the original (gold standard) simulation data as simulated, to the simulated PC-MRI data as measured and to the measurement data after being enhanced by our method as enhanced.
} 


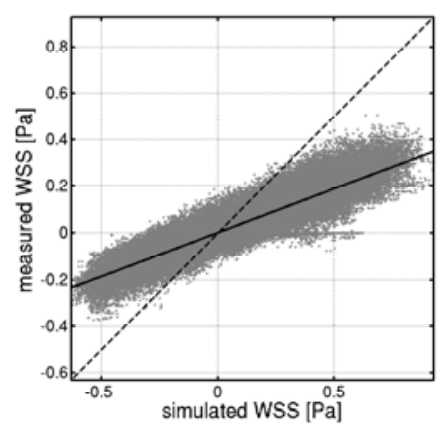

Fig. 2. Correlation of the measured with the simulated WSS

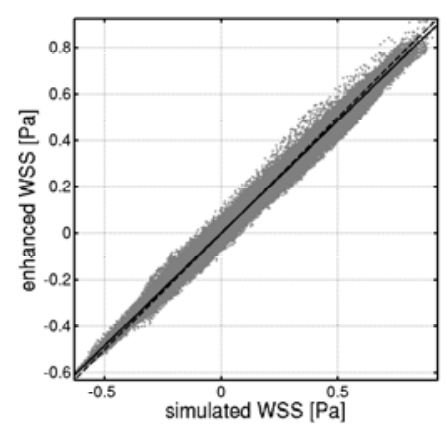

Fig. 3. Correlation of the enhanced with the simulated WSS

is shown, demonstrating that these recirculations are not resolved by the measurement, but do appear correctly in the enhanced flow field. However, despite being sparser and noisier, the measured flow fields are still reasonably accurate, as shown by the statistical data in table 1 .

WSS. Measured, enhanced and simulated WSS distributions at peak systole and end diastole are shown in figures $4(\mathrm{~d})$ and $4(\mathrm{e})$ respectively. One may observe that the WSS as postprocessed from the measurement is clearly underestimated. However, its spatial distribution, which is difficult to perceive directly from this illustration, is approximately correct. Figures 2 and 3 show the statistical correlations between the measured and simulated and between the enhanced and simulated WSS fields respectively for a representative case. It can be seen by the slopes of the regression plots that the absolute values of the measured WSS are significantly smaller than the simulated ones, whereas the enhanced ones are in the correct range. This is mostly due to the partial volume effect which leads to underestimation of the velocities close to the wall. The results were similar in all cases, as shown by the poor slope value for the measured WSS in table 1 The high correlation and slope values obtained by the enhancement method indicate that it is able to reconstruct WSS patterns with very good accuracy and could thus effectively replace full CFD simulations for this task.

Pressure. We show no data for the measured pressure fields, as there is no way of measuring a full pressure field by any known in-vivo, non-invasive method. Despite having no measured pressure information, the reconstructed pressure fields were very well correlated with the simulated ones (see table 1) and the close similarity of the pressure distributions at peak systole and end diastole shown in figures $4(\mathrm{~g})$ and $4(\mathrm{~h})$ is typical for all cases.

\section{Discussion}

The fact that both the measured and enhanced velocities correlate well with the simulated ones confirms the work of other authors (see e.g. [10] or [1]) 


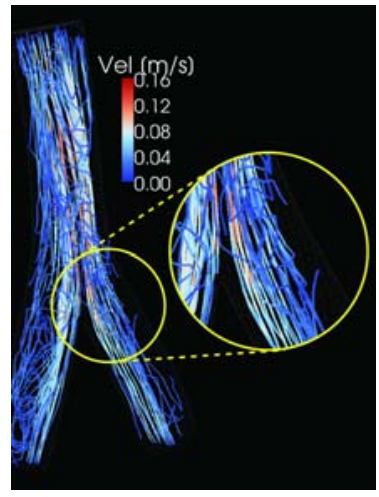

(a) Measured velocity.

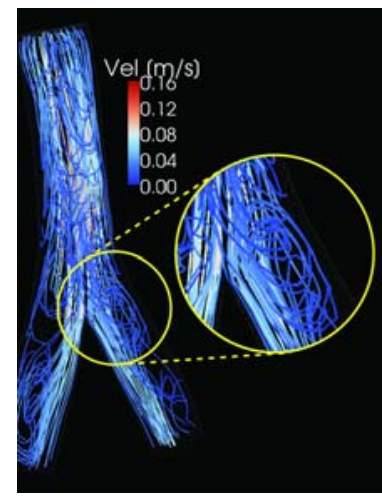

(b) Enhanced velocity.

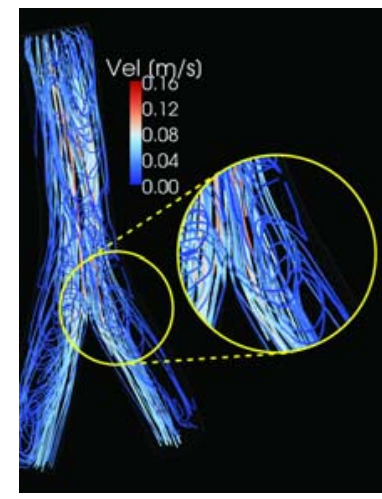

(c) Simulated velocity.

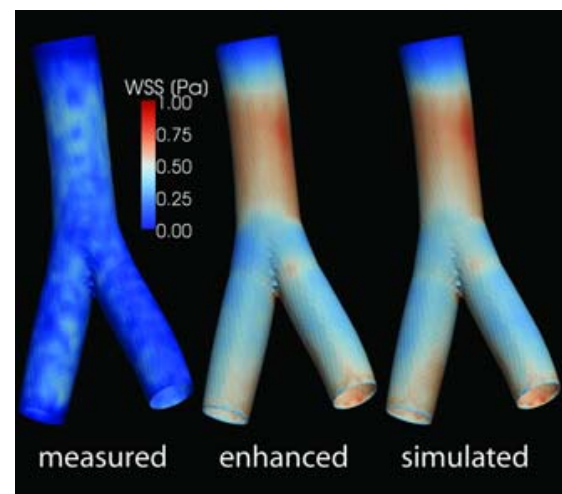

(d) WSS at PS.

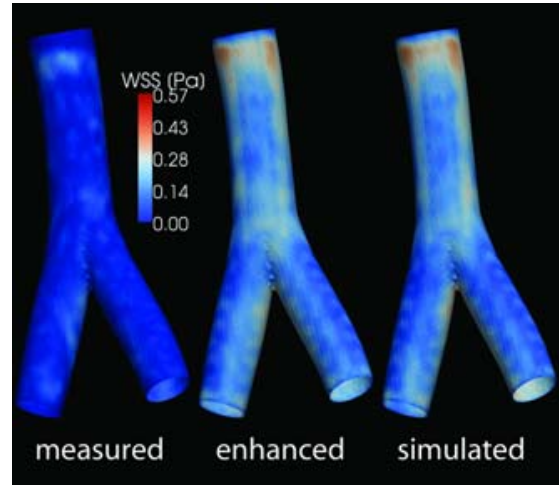

(e) WSS at ED.
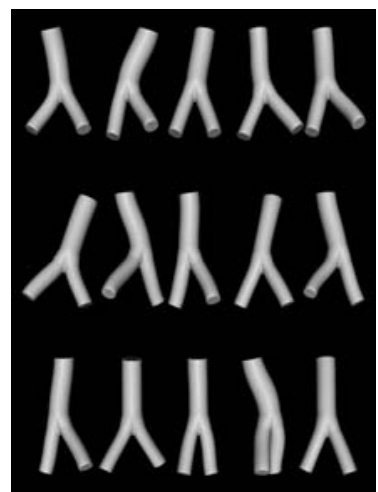

(f) Some geometries.

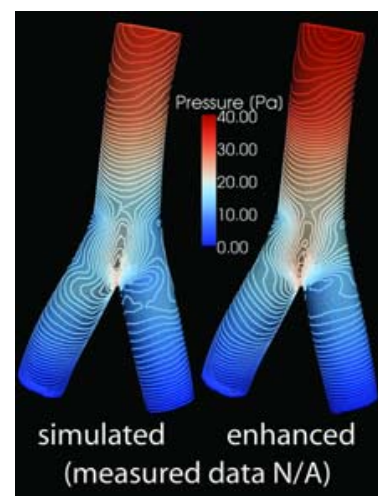

(g) Pressure at PS.

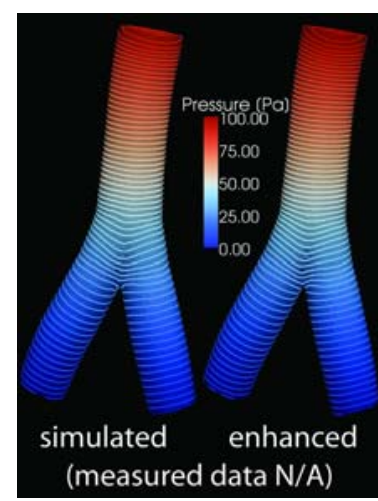

(h) Pressure at ED.

Fig. 4. Streamlines of velocity (top row) at end diastole, with zoom on a recirculation, WSS (middle row) and pressure (bottom row) at both peak systole (PS) and end diastole (ED) and 15 geometries from the database (bottom left) 
who also conclude that PC-MRI is adequate for acquiring the velocity field. However it must be remembered that the enhanced field can have any desired spatio-temporal resolution, whereas the measured PC-MRI data is limited in this respect. Also we focused here on the case of the abdominal aortic bifurcation which is a relatively large vessel. In smaller vessels the resolution limits of this modality will very quickly become prohibitive. However the inability to resolve lower velocities and those near the endothelium has a disastrous effect on the WSS predictions. As shown here and confirmed by others, 12] the WSS values as obtained directly from PC-MRI are by far not accurate enough, in particular with respect to their amplitudes. The approximate location of regions of low or high WSS can be adequately obtained, but only time- and spatially-averaged values can be considered. Should one desire a better quality WSS prediction, information from CFD must be used and our method is able to provide this with high accuracy, without needing the enormous computational resources required for such simulations.

We focused here on two flow quantities other than velocity: WSS and pressure, but obviously any other flow-related variable such as vorticity or oscillatory shear index can also be addressed by this enhancement strategy. Another interesting extension would be the integration of full fluid-structure interaction (FSI). In this study we considered fixed walls, but adding FSI is possible in a straightforward manner. It would require much more computational effort to generate the database but would have little or no impact on the speed of the enhancement procedure while delivering a lot more information such as intramural wall stress.

We focused on healthy, i.e. relatively regular geometries which, while still showing very complex flow patterns, have much less variability than pathological cases such as aneurysms. However, addressing these will not require any modifications of the overall framework, only that the database be adequately larger to account for the added variability.

\section{Conclusions and Outlook}

We have shown a 3D extension of our previously presented flow measurement enhancement strategy and evaluated its performance using a large, representative, synthetic dataset. We were able to demonstrate that it provides reliable predicions about flow and clinically important flow-related quantities, clearly surpassing the quality of direct measurements. We conclude that this is an attractive alternative to costly, fully-blown CFD simulations as it is able to achieve similar quality while being orders of magnitude faster (minutes rather than hours or even days). Future work in this direction will now focus on using real patient data and pathological variability, both to build the database and to test the enhancement. However, this evaluation has shown that our approach is a significant step towards bringing the full richness of CFD into daily clinical practice.

Acknowledgments. This work was supported by the Indo-Swiss Joint Research Programme. 


\section{References}

1. Meckel, S., Stalder, A., Santini, F., Radü, E.W., Rüfenacht, D., Markl, M., Wetzel, S.: In: vivo visualization and analysis of 3-D hemodynamics in cerebral aneurysms with flow-sensitized 4-D MR imaging at 3 T. Neuroradiology 50(6), 473-484 (2008)

2. Tortoli, P., Morganti, T., Bambi, G., Palombo, C., Ramnarine, K.V.: Noninvasive simultaneous assessment of wall shear rate and wall distension in carotid arteries. Ultrasound in Medicine \& Biology 32(11), 1661-1670 (2006)

3. Papathanasopoulou, P., Zhao, S., Köhler, U., Robertson, M.B., Long, Q., Hoskins, P., Xu, X.Y., Marshall, I.: MRI measurement of time-resolved wall shear stress vectors in a carotid bifurcation model, and comparison with CFD predictions. Journal of Magnetic Resonance Imaging 17(2), 153-162 (2003)

4. Steinman, D.A., Vorp, D.A., Ethier, C.R.: Computational modeling of arterial biomechanics: Insights into pathogenesis and treatment of vascular disease. Journal of Vascular Surgery 37(5), 1118-1128 (2003)

5. Sforza, D.M., Putman, C.M., Cebral, J.R.: Hemodynamics of cerebral aneurysms. Annu. Rev. Fluid Mech. 41(1), 91-107 (2009)

6. McGregor, R.H.P., Szczerba, D., von Siebenthal, M., Muralidhar, K., Székely, G.: Exploring the use of proper orthogonal decomposition for enhancing blood flow images via computational fluid dynamics. In: Metaxas, D., Axel, L., Fichtinger, G., Székely, G. (eds.) MICCAI 2008, Part II. LNCS, vol. 5242, pp. 782-789. Springer, Heidelberg (2008)

7. Blanz, V., Vetter, T.: Reconstructing the complete 3D shape of faces from partial information. Informationstechnik und Technische Informatik 44(6), 295-302 (2002)

8. Sun, H., Kuban, B., Schmalbrock, P., Friedman, M.: Measurement of the geometric parameters of the aortic bifurcation from magnetic resonance images. Annals of Biomedical Engineering 22(3), 229-239 (1994)

9. Cheng, C.P., Parker, D., Taylor, C.A.: Quantification of wall shear stress in large blood vessels using lagrangian interpolation functions with cine phase-contrast magnetic resonance imaging. Annals of Biomedical Engineering 30(8), 1020-1032 (2002)

10. Boussel, L., Rayz, V., Martin, A., Acevedo-Bolton, G., Lawton, M.T., Higashida, R., Smith, W.S., Young, W.L., Saloner, D.: Phase-contrast magnetic resonance imaging measurements in intracranial aneurysms in vivo of flow patterns, velocity fields, and wall shear stress: Comparison with computational fluid dynamics. Magnetic Resonance in Medicine 61(2), 409-417 (2009)

11. Hollnagel, D.I., Summers, P.E., Kollias, S.S., Poulikakos, D.: Laser doppler velocimetry (LDV) and 3D phase-contrast magnetic resonance angiography (PCMRA) velocity measurements: Validation in an anatomically accurate cerebral artery aneurysm model with steady flow. Journal of Magnetic Resonance Imaging 26(6), 1493-1505 (2007)

12. Katritsis, D., Kaiktsis, L., Chaniotis, A., Pantos, J., Efstathopoulos, E.P., Marmarelis, V.: Wall shear stress: Theoretical considerations and methods of measurement. Progress in Cardiovascular Diseases 49(5), 307-329 (2007) 